BULL. AUSTRAL. MATH. SOC.

\title{
APPLICATIONS OF CONVERGENCE SPACES
}

\author{
GARY D, RICHARDSON
}

\begin{abstract}
Convergence notions are used extensively in the areas of probability and statistics. Many times proofs can be simplified by considering an appropriate convergence structure on the space and using well-known results from the theory of convergence spaces; for example, compactness arguments are sometimes simplified by using a generalized Ascoli theorem in the convergence space setting. The theory of convergence spaces is also used to generalize some results in probability and statistics.
\end{abstract}

\section{Preliminaries}

Many of the convergence notions studied in probability and statistics are sequential and are sometimes not determined by a topology. Even if they are described by a topology, it is sometimes more convenient to study these notions in the setting of a convergence space. Convergence spaces given here are defined sequentially. Several authors have recently studied convergence spaces from a filter point of view. The necessary definitions and terminology are given below; however, the reader is referred to Novák [11] for further details concerning sequential convergence spaces and Kent [8] for results in the filter setting.

A convergence structure on a set $X$ satisfies the following axioms:

(I) $x_{n} \rightarrow x$ whenever $x_{n}=x, n \geq 1$;

(2) $x_{n_{k}} \rightarrow x$ whenever $x_{n} \rightarrow x$;

Received 3 August 1979. 
(3) $x_{n} \rightarrow x, x_{n}+y$ implies that $x=y$.

The arrow denotes convergence. A convergence structure merely specifies the convergent sequences and limits. The set $X$ along with the convergence structure is called a convergence space. These ideas date back to Fréchet [6].

A point $x$ belongs to the closure of a subset $A$ of $X$ provided there is a sequence in $A$ which converges to $x$. The set of all points of closure of $A$ is denoted by cl $A$. The closure operator in'a convergence space is in general not idempotent. A subset $A$ of $X$ is called closed whenever cl $A=A$. The set of all closed subsets of a convergence space form the closed subsets for a topology on $X$. This associated topological space is denoted by $\lambda X$. A function $f: X \rightarrow Y$ between two convergence spaces is said to be continuous if $f\left(x_{n}\right)+f(x)$ in $y$ whenever $x_{n} \rightarrow x$ in $X$. It is not difficult to show in this case that $f: \lambda X \rightarrow \lambda Y$ is also continuous.

A convergence space is called separable whenever it contains a countable subset $A$ such that $c l A=X$. A subset $A$ is called relatively. compact whenever each sequence in $A$ has a convergent subsequence and compact whenever $A$ is closed and relatively compact.

Let $C(X)\left(C^{*}(X)\right)$ denote the set of all continuous (bounded continuous) real-valued functions from the convergence space $X$ into the real line $R$. Consider the following convergence structure on $C(X): f_{n} \rightarrow f$ iff $f_{n}\left(x_{n}\right) \rightarrow f(x)$ in $R$ whenever $x_{n}+x$ in $X$. For reasons given later, a different convergence structure is defined on $C^{*}(X): f_{n}+f$ iff $f_{n}\left(x_{n}\right) \rightarrow f(x)$ in $R$ whenever $x_{n} \rightarrow x$ in $X$ and also $\left(f_{n}\right)$ is uniformly bounded on $X$. These two convergence spaces are denoted by $C_{c}(X)$ and $C_{c}^{*}(X)$. This type of convergence is sometimes called continuous convergence. It seems to have been first studied by Hahn [7] and later by Cook and Fischer [4] in the filter setting. A subset $H$ of $C_{c}(X)$ is called equicontinuous whenever $f\left(x_{n}\right) \rightarrow f(x)$ uniformly in $f \in H$, provided $x_{n} \rightarrow x$ in $X$. 


\section{The space of probability measures}

The following generalizes the Ascoli theorem to the convergence space setting. The proof is omitted since it resembles the usual one for the separable metric space case.

PROPOSITION 1.1. Let $X$ be a separable convergence space and let $H$ be a subset of $C_{c}(X)$. Then $H$ is relatively compact in $C_{c}(X)$ iff

(1) $H(x)$ is a bounded subset of $R$ for each $x \in X$,

(2) $H$ is equicontinuous.

Let $X$ be a metric space and let $B$ denote the Borel $\sigma$-field; that is, the smallest $\sigma$-field containing the open subsets of $X$. The metric space $X$ is considered to be a convergence space, where the convergent sequences coincide with those determined by the metric. It would be more accurate to say that $X$ is a metrizable convergence space. Let $M(X)$ denote the set of all probability measures defined on $(X, B)$. The convergence structure on $M(X)$ is defined as follows: $P_{n} \rightarrow P$ iff $\int f_{n} d P_{n} \rightarrow \int f d P$ whenever $f_{n} \rightarrow f$ in $C_{c}^{*}(X)$. It can be shown that this satisfies the axioms for a convergence structure, which is the reason for requiring convergent sequences in $C_{c}^{*}(X)$ to be uniformly bounded. Let the set $M(X)$ with this convergence structure be denoted by $M_{c}(X)$. Note that the map $\omega: M_{c}(X) \times C_{c}^{*}(X) \rightarrow R$, defined by $\omega(p, f)=\int f d P$, is jointly continuous. Another desirable property is the following.

PROPOSITION 1.2. Let $X$ be a metric space. Then the map $\phi: M_{c}(X) \rightarrow C_{c}\left(C_{c}^{*}(X)\right)$, defined by $\phi(P)(f)=\int f d P$, is a closed embedding.

Proof. If $P \in M(X)$, then by the dominated convergence theorem, $\phi(P): C_{c}^{*}(X) \rightarrow R$ is continuous, and so $\phi(P) \in C\left(C_{c}^{*}(X)\right)$. Let $\phi(P)=\phi(Q)$; then $\int f d P=\int f d Q$ for each $f \in C^{*}(X)$ and it follows (for example, see Billingsley [2], Theorem 1.3) that $P=Q$. Hence $\phi$ is one-to-one. The continuity of $\phi$ and $\phi^{-1}$ follows easily from the definitions and so $\phi$ is an embedding. 
Suppose that $\phi\left(P_{n}\right) \rightarrow h$ in $C_{c}\left(C_{c}^{*}(X)\right)$. Let $\left(f_{n}\right)$ be a sequence in $C^{*}(X)$ such that $f_{n} \downarrow 0$; then by a theorem of Dini, $f_{n} \rightarrow 0$ in $C_{c}^{*}(X)$, and since $h$ is continuous, $h\left(f_{n}\right) \rightarrow h(0)=0$ in $R$. Hence by the Daniell representation theorem (for example, see Ash [1], 4.2.9, p. 175) there is a $P \in M(X)$ such that $\phi(P)=h$ and so the range of $\phi$ is a closed subspace of $C_{C}\left(C_{c}^{*}(X)\right)$. / /

A study of continuous convergence in $C(X)$ in the filter setting is given by Feldman [5], for $X$ a convergence space in the filter sense; however, one must be careful in translating these results to the sequential setting. The following can be deduced from Feldman [5, Theorem 3]. Let $X$ be a separable metric space; then $c_{c}(X)$ is a separable convergence space. It can be shown from this that $C_{c}^{*}(X)$ is also a separable convergence space.

Recall that a subset $H$ of $M(X)$ is called tight if for each $\varepsilon>0$ there is a compact subset $K$ of $X$ such that $P(K)>1-\varepsilon$ whenever $P \in H$. Proposition 1.1 provides a straightforward method for proving compactness for many spaces in probability and statistics.

PROPOSITION 1.3 (Prohorov). Let $X$ be a separable metric space and let $H$ be a tight subset of $M(X)$. Then $H$ is a relatively compact subset of $M_{c}(X)$.

Proof. From Proposition 1.2, it suffices to show that $\phi(H)$ is a relatively compact subset of $C_{c}\left(C_{c}^{*}(X)\right)$. Let $f \in C^{*}(X)$; then $\phi(H)(f)$ is a bounded subset of $R$. Suppose that $f_{n}+f$ in $C_{c}^{*}(X)$ and $\left|f_{n}\right| \leq M, n \geq 1$. Given $\varepsilon>0$, choose a compact subset $K$ of $X$ such that $P(X-K)<\varepsilon / 4 M$ for each $P \in H$. Since $f_{n} \rightarrow f$ uniformly on $K$, then let $n_{0}$ be such that $\left|f_{n}(x)-f(x)\right|<\varepsilon / 2$ for each $x \in K, n \geq n_{0}$. Then

$$
\left|\int f_{n} d P-\int f d P\right| \leq \int_{K}\left|f_{n}-f\right| d P+\int_{X-K}\left|f_{n}-f\right| d P<\varepsilon
$$

for each $P \in H, n \geq n_{0}$. It follows that $\phi(H)$ is an equicontinuous subset of $C_{c}\left(C_{c}^{*}(X)\right)$. From the previously mentioned result that $C_{c}^{*}(X)$ 
is a separable convergence space, it follows by Proposition 1.1 that $\phi(H)$ is relatively compact. //

Let $M_{\omega} X$ denote the usual weak convergence on $M(X)$; that is, $P_{n} \rightarrow P$ iff $\int f d P_{n}+\int f d P$ for each $f \in C^{*}(X)$.

PROPOSITION 1.4. Let $X$ be a metric space; then $M_{\omega} X=M_{c} X$.

Proof. Certainly continuous convergence implies weak convergence. Conversely, let $P_{n} \rightarrow P$ in $M_{\omega} X$ and let $f_{n} \rightarrow f$ in $C^{*}(X)$ with $\left|f_{n}\right| \leq M$. Let $Q_{n}=P P_{n} f_{n}^{-1}$ and $Q=P f^{-1}$ be the induced probability measures on $(R, B)$. Then from Billingsley [2, Theorem 5.5, p. 34], $Q_{n}+Q$ in $M_{\omega} R$. Since $\left(f_{n}\right)$ is uniformly bounded, then the identity function on $R$ is uniformly integrable re $\left(Q_{n}\right)$, so $\int x d Q_{n} \rightarrow \int s d Q$ (for example, see Lòve $[10, \mathrm{p} .183])$. Hence $\int f_{n} d P_{n} \rightarrow \int f d P$, so $P_{n} \rightarrow P$ in $M_{c}(X) \cdot \quad / /$

\section{The space of test functions}

The domain for a test in statistics is generally taken to be a Euclidean subspace; however, our discussion is relative to the metric space setting. Let $X$ denote a metric space and $B$ the $\sigma$-field generated by all the open subsets of $X$. Let $T$ denote the set of all tests on $(X, B)$; that is, $\phi \in T$ provided $\phi: X \rightarrow[0,1]$ is a measurable function on $(X, B)$.

Let $\mu$ be a $\sigma$-finite measure on $(X, B)$ and let $Y$ denote the set of all $\mu$-integrable functions on $(X, B)$. Define convergence in $Y$ to be $L^{\prime}$-convergence; that is, $f_{n} \rightarrow f$ iff $\int\left|f_{n}-f\right| d \mu \rightarrow 0$. More precisely, points in $Y$ are equivalence classes and $Y$ is a metric space. Define the following convergence structure in $T: \phi_{n} \rightarrow \phi$ iff $\int \phi_{n} f d \mu \rightarrow \int \phi f d \mu$ for each $f$ in $Y$. Let $T$ with this convergence structure be denoted by $T_{\mu}$. The space $T_{\mu}$ is compact whenever $X$ is separable (for example, see Lehmann [9, Theorem 3, p. 354]) and forms the 
basis for proving the existance of certain optimal test in statistics. Proposition 1.1 provides a straightforward alternative method for showing that $T_{\mu}$ is compact. As in the case for $Y$, points in $T_{\mu}$ are actually equivalence classes; that is, $\phi \sim \psi$ iff $\phi=\psi$ almost everywhere [ $\mu]$, or equivalently, $\int \phi f d \mu=\int \psi f d \mu$ for each $f \in Y$.

PROPOSITION 2.1. Let $X$ be a metric space and $\mu$ a o-finite measure on $(X, B)$. The map $\alpha: T_{\mu} \rightarrow C_{c}(y)$, defined by $\alpha(\phi)(f)=\int f \phi d \mu$, is a closed embedding.

Proof. The fact that $\alpha$ is an embedding is routine to check. Suppose that $\alpha\left(\phi_{n}\right) \rightarrow h$ in $C_{c}(y) ;$ then $h(f)=\underset{n}{\lim } \int f \phi_{n} d \mu$ for each $f \in Y$. Hence $h$ is a bounded linear functional on $y$ and from the Riesz representation theorem there is a $g \in L^{\infty}$ such that $h(f)=\int f g d \mu$ for each $f \in Y$. It is easy to show that $0 \leq g \leq 1$ almost everywhere [ $\mu]$, so $g \in T_{\mu}$ and hence $h=\alpha(g)^{\circ}$. Thus $\alpha\left(T_{\mu}\right)$ is a closed subspace of $C_{c}(Y) \cdot \quad / /$

A straightforward application of Propositions 1.1 and 2.1 gives an alternative proof of the following result.

PROPOSITION 2.2. Let $X$ be a separable metric space and $\mu$ a o-finite measure on $(X, B)$. Then the space $T_{\mu}$ is compact.

Let us consider an appropriate test space whenever $P \subset M(X)$. Again let $T$ denote the set of all tests on $(X, B)$ and define $\phi \sim \psi$ iff $\int \phi d P=\int \psi d P$ for each $P \in P$. This is an equivalence relation in $T$ and two tests are equivalent whenever they have the same power functions on $P$. The set of equivalence classes with the following convergence structure is denoted by $T_{p}: \phi_{n}+\phi$ iff for each $P \in P$, $\int \phi_{n} d P \rightarrow \int \phi d P$. If $\mu$ is a $\sigma$-finite measure on $(X, B)$ and $P=\{P \in M(X) \mid P \ll \mu\}$, then it is not difficult to show that $T_{\mu}=T_{p}$. Hence $T_{p}$ seems to be a proper generalization of $T_{\mu}$. The following fact 
about $T_{\mu}$ is useful in investigating properties of $T_{p}$.

PROPOSITION 2.3. Let $X$ be a separable metric space and $\mu$ a $\sigma$-finite measure on $(X, B)$. Then the convergence space $T_{\mu}$ is metrizable.

Proof. Since $X$ is a separable metric space, then $Y$, defined above, is also separable. Let $A=\left\{g_{1}, g_{2}, \ldots\right\}$ be a countable dense subset of $Y$ and let $R^{\infty}$ denote a countably infinite product of $R$ with convergence structure of pointwise convergence of sequences. Define $\alpha: T_{\mu}+R^{\infty}$ by $\alpha(\phi)(n)=\int \phi g_{n} d \mu, n \geq 1$. Then it is easy to show that $\alpha$ is an embedding and hence $T_{\mu}$ is metrizable. //

The notation $P \ll \mu$ denotes the fact that $P$ is dominated by the $\sigma$-finite measure $\mu$. Suppose that $\left(\phi_{n}\right)$ fails to converge to $\phi$ in $T_{p}$. Then there is a $P \in P$ such that $\int \phi_{n} d P \rightarrow \int \phi d P$. Let $h: T_{p} \rightarrow R$ be defined by $h(\psi)=\int \psi d P$. Then $h$ is continuous and $h\left(\phi_{n}\right) \rightarrow h(\phi)$ in $R$. This implies that the convergent sequences of the completely regular topology on $T_{p}$ generated by the continuous functions coincide with the convergent sequences in $T_{p}$. In the language of Novák [11], $T_{p}$ is a sequentially regular convergence space. In particular, $T_{p}$ and $\lambda T_{p}$ have the same convergent sequences, where $\lambda T_{p}$ is the topological space whose closed sets are precisely those closed in $T_{p}$; that is, $\mathrm{cl}_{T_{p}} A=A$

PROPOSITION 2.4. Let $X$ be a separable metric space. If $P \subset M(X)$ and $P \ll \mu$, then $T_{p}$ is compact and metrizable.

Proof. Since each equivalence class re $\mu$ is contained in the corresponding equivalence class re $\mathrm{P}$, then let $j: T_{\mu} \rightarrow T_{p}$ denote the natural map. Since $j$ is continuous, then by Proposition 2.2, $T_{p}$ is compact. Also $j: \lambda T_{\mu}+\lambda T_{p}$ is continuous and moreover since $T_{\mu}$ is compact and metrizable it follows easily that $j: \lambda T_{\mu} \rightarrow \lambda T_{p}$ is a 
topological quotient map. It is known that a Hausdorff quotient of a compact metrizable space is metrizable (for example, see Bourbaki [3, Proposition 17, p. 159]) and since $T_{p}$ and $\lambda T_{p}$ agree on convergent sequences, $T_{p}$ is metrizable. //

Let $X, Y$ be metric spaces with corresponding Borel $\sigma$-fields and let $\tau: X \rightarrow Y$ be a measurable function. If $P \subset M(X)$, then let $T_{X}$ denote the test space re $P$ and let $T_{Y}$ be the test space re $P_{\tau}{ }^{-1}$.

PROPOSITION 2.5. Let $\tau: X \rightarrow Y$ be a measurable function, where $X$ and $Y$ are metric spaces. Let $P \subset M(X)$ and let $\alpha: T_{Y} \rightarrow T_{X}$ be defined by $\alpha(\psi)=\psi \circ \tau$. Then $\alpha$ is an embedding and moreover, if $\tau$ is a sufficient statistic for $P$, then $a$ is an onto embedding.

Proof. Suppose that $\alpha\left(\psi_{1}\right)=\alpha\left(\psi_{2}\right)$ in $T_{X}$; that is $\int \psi_{1} \circ \tau d P=\int \psi_{2} \circ \tau d P$ for $P \in P$. Then $\int \psi_{1} d Q=\int \psi_{2} d Q$ for each $Q=P \tau^{-1}$, so $\psi_{1}=\psi_{2}$ in $T_{Y}$; that is, $\psi_{1}$ and $\psi_{2}$ belong to the same equivalence class. Hence $\alpha$ is one-to-one. The continuity of $\alpha$ and $\alpha^{-1}$ are easy to show and so $\alpha$ is an embedding. Suppose that $\tau$ is a sufficient statistic for $P$ and let $\phi \in T_{X}$. Let $\psi=E(\phi \mid \tau)$; then $\psi \in T_{Y}$. Furthermore, for $Q=P \tau^{-1}, P \in P$,

$$
\int(\psi \circ \tau) d P=\int \psi d Q=\int \phi d P
$$

and hence it follows that $\psi \circ \tau=\phi$ in $T_{X}$. Thus $\alpha(\psi)=\phi$ and so $\alpha$ is an onto embedding. //

Let us consider a convergence structure for $P \subset M(X)$ which has properties desirable for hypothesis testing. It seems desirable to have joint continuity of the map $\omega: T_{p} \times P \rightarrow[0,1]$ defined by $\omega(\phi, P)=\int \phi d P$. This, in particular, implies that the power function for each test is continuous on $P$. This leads us to define the following convergence structure on $P: P_{n} \rightarrow P$ iff for each $\phi_{n} \rightarrow \phi$ in $T_{p}$, 
$\int \phi_{n} d P_{n} \rightarrow \int \phi d P$. The set $P$ equipped with this convergence structure is denoted by $P_{c}$. In fact, this is the coarsest convergence structure on $P$ such that the map $\omega$ is jointly continuous.

Let $\tau: X \rightarrow Y$ be a measurable function between two metric spaces and let $P \subset M(X)$. Since $P_{\tau}^{-1} \subset M(Y)$, then convergence in $P_{\tau}^{-1}$ is defined in a similar manner as is given for $P \subset M(X)$. Let $P_{\tau}^{-1}$ with this convergence.structure be denoted by $P_{c}^{*}$. If $\alpha: P_{c}+P_{c}^{*}$ denotes the map $\alpha(P)=P \tau^{-1}$, then it follows easily that $\alpha$ is continuous.

PROPOSITION 2.6. Let $\tau: X \rightarrow Y$ be a sufficient statistic for $P \subset M(X)$, where $X$ and $Y$ are metric spaces. Then $\alpha: P_{c} \rightarrow P_{c}^{*}$, defined above, is an onto embedding.

Proof. Let $P_{1}, P_{2} \in P$ such that $Q_{1}=\alpha\left(P_{1}\right)=\alpha\left(P_{2}\right)=Q_{2}$. If $A \in B$, then $\phi=I_{A} \in T_{X}$ and so $\psi=E(\phi \mid \tau) \in T_{Y}$. Hence

$$
P_{1}(A)=\int \phi d P_{1}=\int \psi d Q_{1}=\int \psi d Q_{2}=\int \phi d P_{2}=P_{2}(A)
$$

so $P_{1}=P_{2}$ and $\alpha$ is one-to-one. The continuity of $\alpha$ holds as mentioned above. Suppose that $Q_{n}=P_{n} \tau^{-1} \rightarrow P \tau^{-1}=Q$ in $P_{c}^{*}$ and let $\phi_{n} \rightarrow \phi$ in $T_{X}$. If $\psi_{n}=E\left(\phi_{n} \mid \tau\right)$ and $\psi=E(\phi \mid \tau)$, then it follows easily that $\psi_{n} \rightarrow \psi$ in $T_{Y}$ and hence $\int \psi_{n} d Q_{n} \rightarrow \int \psi d Q$, or $\int \phi_{n} d P_{n} \rightarrow \int \phi d P$. Hence $P_{n} \rightarrow P$ in $P_{c}$ and so $\alpha$ is an embedding. // Let $\tau: X \rightarrow Y$ be a measurable function and $\alpha: P_{c} \rightarrow P_{c}^{*}$ defined as above. If $\psi \in T_{Y}$ and is $\delta$-similar on the boundary for testing $P_{0} \tau^{-1}$ vs $P_{1} \tau^{-1}$, where $P_{0} \cup P_{1}=P$ and $P_{0} \cap P_{1}=\phi$, then $\phi=\psi 0^{\circ} \tau$ is also $\delta$-similar on the boundary for testing $P_{0}$ vs $P_{1}$. The boundary for testing $P_{0}$ vs $P_{1}$ is defined to be the set cl $P_{0} \cap c l P_{1}$ in the convergence space $P_{c}$. The above follows from the continuity of the map 
$\alpha: P_{c} \rightarrow P_{c}^{*}$. Moreover, since each power function $B_{\phi}: P_{c}+[0,1]$ is continuous, then from Lehmann [9, Lemma 1, p. 126] a UMP $\delta$-similar on the boundary test of size $\delta$ is UMP unibased of size $\delta$.

The next result follows from Proposition 2.6.

COROLLARY 2.7. Let $\tau: X \rightarrow Y$ be a sufficient statistic for $P \subset M(X)$, where $X$ and $Y$ are metric spaces. Let $\psi$ be a UMP $\delta$-simizar on the boundary test of size $\delta$ for testing $P_{0} \tau^{-1}$ vs $P_{1} \tau^{-1}$. Then $\psi \circ \tau$ is a UMP $\delta$-simizar on the boundary test of size $\delta$ for testing $P_{0}$ vs $P_{1}$.

\section{The space $P_{c}$}

Let $X$ be a metric space, $B$ the corresponding Borel $\sigma-f i e l d$, and $P \subset M(X)$. Then for $P, Q \in P, d(P, Q)=\sup _{A \in B}|P(A)-Q(A)|$ defines a metric on $P$. Let $P$ equipped with the convergence structure determined by the metric be denoted by $P_{d}$. Note that $P_{n} \rightarrow P$ in $P_{d}$ iff $P_{n}(A) \rightarrow P(A)$ uniformly in $A \in B$. Lehmann [9, p. 352] shows that whenever $P \ll \mu, P_{n} \rightarrow P$ in $p_{d}$ iff $\int\left|p_{n}-p\right| d \mu \rightarrow 0$, where $p_{n}, p$ are the probability densities re $\mu$. Let $P_{\omega}$ denote the subspace inherited from $M_{\omega} X$, where $\omega$ denotes weak convergence of probability measures.

PROPOSITION 3.1. Let $X$ be a metric space and $P \subset M(X)$. Then $P_{d} \geq P_{c} \geq P_{\omega}$ and when $T_{p}$ compact, $P_{d}=P_{c}$; in particular, $P_{d}=P_{c}$ whenever $P \ll \mu$ and $X$ is separable.

Proof. Suppose that $P_{n} \rightarrow P$ in $P_{d}$ and $\phi_{n} \rightarrow \phi$ in $T_{p}$. Let $\mu$ be a o-finite measure which dominates $P_{n}, P$, all $n \geq 1$. If $p_{n}, p$ are densities re $\mu$, then by the above remark $\int\left|p_{n}-p\right| d \mu \rightarrow 0$. It follows easily by using the triangle inequality that $\int \phi_{n} d P_{n} \rightarrow \int \phi d P$, so $P_{n} \rightarrow P$ in $P_{c}$ and $P_{d} \geq P_{c}$.

Let $P_{n} \rightarrow P$ in $P_{c}$ and $f \in C^{*}(X),|f| \leq M$. Then $\int f d P_{n} \rightarrow \int f d P$ 
follows by decomposing $f$ into $f^{+}$and $f^{-}$, so $P_{n} \rightarrow P$ in $P_{\omega}$ and $P_{c} \geq P_{\omega}$

Suppose that $T_{p}$ is compact and $P_{n} \rightarrow P$ in $P_{c}$. If there exists an $\varepsilon>0$ and a sequence $\left(A_{n}\right)$ in $B$ such that $\left|P_{n}\left(A_{n}\right)-P\left(A_{n}\right)\right| \geq \varepsilon$ for each $n \geq 1$, then let $\phi_{n}=I_{A_{n}}$, so $\phi_{n_{k}} \rightarrow \phi$ in $T_{p}$. Hence $\left|P_{n_{k}}\left(A_{n_{k}}\right)-\int \phi d P\right|<\varepsilon / 2$ and $\left|P\left(A_{n_{k}}\right)-\int \phi d P\right|<\varepsilon / 2$ for $k$ sufficiently large. Thus, for $k$ sufficiently large, $\left|P_{n_{k}}\left(A_{n_{k}}\right)-P\left(A_{n_{k}}\right)\right|<\varepsilon$, which contradicts the above. This argument shows that $P_{n} \rightarrow P$ in $P_{d}$ and so $P_{d}=P_{c}$. The last part of the proposition follows from Proposition 2.4.//

PROPOSITION 3.2. Let $X$ be a separable metric space and let $P \subset M(X)$. Then the following are equivalent:

(1) $T_{p}$ is separable and metrizable;

(2) $P_{c}$ is separable;

(3) $P \ll \mu$;

(4) $T_{p}$ is compact and metrizable;

(5) $\lambda T_{p}$ is a second countable topological space;

(6) $P_{c}$ is separable and metrizable.

Proof. (1) $\Rightarrow(2)$. It is straightforward to verify that the map $\alpha: P_{c}+C_{c}\left(T_{p}\right)$, defined by $\alpha(P)(\phi)=\int \phi d P$, is an embedding. Hence from Feldman [5, Theorems 3 and 5], it follows that $P_{c}$ is separable.

(2) $\Rightarrow(3)$. The argument given in Lehmann [9, p. 353] applies here.

$(3) \Rightarrow(4)$. This follows from Proposition 2.4 .

$(4) \Rightarrow(5)$. It follows since $\lambda T_{p}$ is compact, metrizable and hence second countable.

$(5) \Rightarrow(6)$. Since the map a above is an embedding, then from feldman 
[5, Theorems 3 and 5], $P_{c}$ is separable. This implies from the above that $P \ll \mu$ and so by Proposition 3.1, $P_{c}=P_{d}$ is metrizable.

$(6) \Rightarrow(1)$. Since $P \ll \mu$, then from Proposition 2.4, $T_{p}$ is compact, metrizable and hence separable. //

Let $X$ be a metric space, $P \subset M(X)$ and consider the problem of teating $P_{0}$ vs $P_{1}$ at level $\alpha$, where $P_{0} \cup P_{1}=P$ and $P_{0} \cap P_{1}=\phi$. Lehmann [9, p. 340] defines the envelope power function, $B^{*}$, on $P$ by $\beta^{*}(P)=\sup _{\phi \in T_{\alpha}} \int \phi d P$, where $T_{\alpha}=\left\{\phi \in T_{p} \mid \phi\right.$ is a level $\alpha$ test $\}$. Nöte that $T_{\alpha}$ is a closed subspace of $T_{p}$ and hence is compact whenever the latter is. The existence of many optimal tests in the dominated case is based on the fact that $T_{\mu}$ is compact, where $p \ll \mu$. Similar results may be expected to hold in the general case whenever $T_{p}$ is assumed to be compact.

Let $\gamma: T_{p} \rightarrow C_{c}\left(P_{c}\right)$ be defined by $\gamma(\phi)(P)=\int \phi d P$. Then it follows easily that $\gamma$ is an embedding. Hence $T_{\alpha}$ equicontinuous means that $Y\left(T_{\alpha}\right)$ is an equicontinuous subset of $C_{c}\left(P_{c}\right)$. Similarly, $P_{1}$ equicontinuous means that $\alpha\left(P_{1}\right)$ is an equicontinuous subset of $C_{c}\left(T_{p}\right)$.

PROPOSITION 3.3. Let $X$ be a metric space and $P \subset M(X)$. If $T_{\alpha}$ is equicontinuous, then $B^{*}$ is a continuous function on $P_{C}$.

Proof. Let $P_{n} \rightarrow P$ in $P_{c}$. Since

$$
\sup _{\phi \in T_{\alpha}} \int \phi d P_{n} \leq \sup _{\phi \in T_{\alpha}}\left|\int \phi d P_{n}-\int \phi d P\right|+\sup _{\phi \in T_{\alpha}} \int \phi d P
$$

then by symmetry, it follows that

$$
\left|B^{*}\left(P_{n}\right)-B^{*}(P)\right| \leq \sup _{\phi \in T_{\alpha}}\left|\int \phi d P_{n}-\int \phi d P\right| .
$$

Since $T_{\alpha}$ is equicontinuous, then it follows that $B^{*}\left(P_{n}\right) \rightarrow B^{*}(P)$. // 
Define the function $\delta: T_{\alpha} \rightarrow[0,1]$ for the above testing problem by $\delta(\phi)=\sup _{P \in P_{1}}\left(\beta^{*}(P)-\int \phi d P\right)$. A test making $\delta$ a minimum is called most stringent of Zevel a (for example, see Lehmann [9, p. 340]).

PROPOSITION 3.4. Let $X$ be a metric space and $P \subset M(X)$. If $P_{1}$ is equicontinuous, then $\delta$ is a continuous function on $T_{\alpha}$.

Proof. Let $\phi_{n} \rightarrow \phi$ in $T_{\alpha}$. Since

$$
\sup _{P \in P_{1}}\left(\beta^{*}(P)-\int \phi d P\right) \leq \sup _{P \in P_{1}}\left(\beta^{*}(P)-\int \phi_{n} d P\right)+\sup _{P \in P_{1}}\left|\int \phi_{n} d P-\int \phi d P\right| \text {, }
$$

then by symmetry,

$$
\left|\delta(\phi)-\delta\left(\phi_{n}\right)\right| \leq \sup _{P \in P_{1}}\left|\int \phi_{n} d P-\int \phi d P\right|
$$

Since $P_{1}$ is equicontinuous, then it follows that $\delta\left(\phi_{n}\right) \rightarrow \delta(\phi)$. //

COROLLARY 3.5. Let $X$ be a metric space and $P \subset M(X)$. If $T_{\alpha}$ is compact and $P_{1}$ is equicontinuous, then there exists a most stringent test of level $\alpha$.

Moreover, it is not difficult to prove the following more general result.

PROPOSITION 3.6. Let $X$ be a metric space and $P \subset M(X)$. Suppose that $P_{1}=c 1 P_{1}^{*}$, where $P_{1}^{*}=\bigcup_{n=1}^{\infty} p^{n}, p^{n} \subset p^{n+1}$ and $p^{n}$ is equicontinuous, $\alpha 2 l n \geq 1$. If $T_{\alpha}$ is compact, then there exists a most stringent test of level $\alpha$.

If $P \ll \mu$ and $X$ is a separable metric space, then $T_{p}$ is compact and $P_{c}$ is separable and so Proposition 3.6 implies that a most stringent test of level $\alpha$ exists for this case. 


\section{Weakly uniformly integrable functions}

The key to the proof of Proposition 1.4 is the fact that if $P_{n} \rightarrow P$ in $M_{\omega} X$ and $g$ is a continuous real-valued function on $X$ which is uniformly integrable re $\left(P_{n}\right)$, then $\int g d P_{n} \rightarrow \int g d P$. This section is devoted to giving a converse of this result, which is sometimes useful in showing that a particular convergence structure on $M(X)$ coincides with weak convergence.

PROPOSITION 4.1. Let $X$ be a metric space and suppose that $P_{n} \rightarrow P$ in $M_{\omega} X$ and $g$ is a continuous real-valued function on $X$ which is $P_{n}, P$ integrable for each $n \geq 1$. Then $\int g d P_{n} \rightarrow \int g d P$ iff

$$
\lim _{c \rightarrow \infty} \overline{\lim }_{n \rightarrow \infty}\left|\int_{[|g| \geq c]} g d P\right|=0 \text {. }
$$

Proof. Suppose that $\int g d P_{n} \rightarrow \int g d P$. Since $g$ is P-integrable, for $\varepsilon>0$, choose $c_{0}>0$ such that $\int_{\left[|g| \geq c_{0}\right]}|g| d P<\varepsilon$. Let $c \geq c_{0}$ and $I=[-c, c), J=(-c, c]$.

Since $g \cdot 1 g^{-1}(I)$ is lower semicontinuous on $X$, then by Ash [ 1 , Theorem 4.5.1, p. 196],

$$
\frac{\lim }{n} \int_{g^{-1}(I)} g d P\left(\geq \int_{g^{-I}(I)} g d P\right.
$$

Hence

$$
\begin{aligned}
\frac{\lim }{n} \int_{g^{-1}(I)} & g d P_{n}+\overline{\lim } \int_{X-g^{-1}(I)} g d P_{n} \leq \overline{\lim } \int g d P_{n}=\int g d P \\
& =\int_{g^{-1}(I)} g d P+\int_{X-g^{-1}(I)} g d P \leq \frac{\lim }{n} \int_{g^{-1}(I)} g d P_{n}+\int_{X-g^{-1}(I)} g d P
\end{aligned}
$$

and so it follows that

$$
\overline{\lim } \int_{X-g^{-1}(I)} g d P_{n} \leq \int_{X-g^{-1}(I)} g d P<\varepsilon .
$$


Since

$$
\int_{g^{-1}(-c)} g d P_{n} \leq 0
$$

then it follows that

$$
\overline{\lim }_{n} \int_{[|g| \geq c]} g d P_{n}<\varepsilon \text {. }
$$

A similar argument using $J$ shows that

$$
\frac{\lim }{n} \int_{[|g| \geq c]} g d P_{n}>-\varepsilon ;
$$

hence

$$
\varlimsup_{n}^{\lim }\left|\int_{[|g| \geq c]} g d P_{n}\right|<\varepsilon
$$

for each $c \geq c_{0}$ and so

$$
\lim _{c \rightarrow \infty} \overline{\lim }_{n}\left|\int_{[|g| \geq c]} g d P_{n}\right|=0 .
$$

The converse follows easily by using the transformation theorem in conjunction with the Helley-Bray lemma. //

Let us call a sequence $\left(f_{n}\right)$ of continuous real-valued functions on a metric space $X$ weakly uniformly integrable re a sequence of probability measures $\left(P_{n}\right)$ on $M(X)$ if each $f_{n}$ is $P_{n}$ integrable, $n \geq 1$, and

$$
\lim _{c \rightarrow \infty} \varlimsup_{n \rightarrow \infty}\left|\int_{\left[\left|f_{n}\right| \geq c\right]} f_{n} d P_{n}\right|=0 .
$$

From Proposition 4.1, along with the transformation theorem, the following result is obtained.

COROLLARY 4.2. Let $X$ be a metric space and let $\left(f_{n}\right)$ be a sequence of continuous real-valued functions on $X$. Suppose that $P_{n} \rightarrow P$ in $M_{\omega} X$ and each $f_{n}$ is $P_{n}$-integrable for each $n \geq 1$. If $f_{n} \rightarrow f$ in $c_{c}(X)$, then $\int f_{n} d P_{n} \rightarrow \int f d P$ iff $\left(f_{n}\right)$ is weakly uniformly integrable 
re $\left(P_{n}\right)$

Proposition 1.4 could have been proved by using Corollary 4.2 since if $f_{n} \rightarrow f$ in $C_{c}^{*}(X)$, then $\left(f_{n}\right)$ is uniformly bounded and hence uniformly integrable re $\left(P_{n}\right)$. One can easily give examples of weakly uniformly integrable sequences which are not uniformly integrable with respect to a sequence of probability measures.

\section{References}

[1] Robert 8. Ash, Real conalysis and probability (Probability and Mathematical Statistics, 11. Academic Press, New York and London, 1972).

[2] Patrick Billingsley, Convergence of probability measures (John Wiley \& Sons, New York, London, Sydney, Toronto, 1968).

[3] Nicolas Bourbaki, Elements of mathematics. General topology, Part 2 (Hermann, Paris; Addison-Wesley, Reading, Massachusetts; 1966).

[4] C.H. Cook and H.R. Fischer, "On equicontinuity and continuous convergence", Math. Ann. 159 (1965), 94-104.

[5] William A. Feldman, "Axioms of countability and the algebra $C(X)$ ", Pacific J. Math. 47 (1973), 81-89.

[6] Maurice Fréchet, "Sur quelques points du calcul fonctionnel", Rend. Circ. Mat. Palermo 22 (1906), 1-74.

[7] H. Hahn, Theorie der reellen Funktionen, Erster Band (J. Springer, Berlin, 1921).

[8] D.C. Kent and G.D. Richardson, "Minimal convergence spaces", Trans. Amer. Math. Soc. 160 (1971), 487-499.

[9] E.L. Lehmann, Testing statistical hypotheses (John Wiley \& Sons, New York; Chapman \& Hall, London; 1959).

[10] Michel Loève, Probability theory, and edition (Van Nostrand, Princeton, New Jersey; Toronto; New York; London; 1960). 
[11] Josef Novák, "On convergence spaces and their sequential envelopes", Caechoslovak Math. J. 15 (90) (1965), 74-100.

Department of Mathematics,

East Carolina University;

Greenville,

North Carolina 27834,

USA. 